\title{
Analysis of the value and correlation of IGF-1 with GH and IGFBP-3 in the diagnosis of dwarfism
}

\author{
YUN WANG $^{1}$, HE ZHANG $^{2}$, MENG CAO $^{3}$, LINGFENG KONG $^{2}$ and XIAOLI GE ${ }^{1}$ \\ ${ }^{1}$ Department of Pediatrics, The Affiliated Wuxi No. 2 People's Hospital of Nanjing Medical University, \\ Wuxi, Jiangsu 214000; Departments of ${ }^{2}$ Pediatrics and ${ }^{3}$ Endocrinology, The First Affiliated Hospital \\ of Xinxiang Medical University, Xinxiang, Henan 453000, P.R. China
}

Received November 8, 2018; Accepted February 27, 2019

DOI: $10.3892 /$ etm.2019.7393

\begin{abstract}
Correlation between the value of insulin-like growth factor-1 (IGF-1) in the diagnosis of dwarfism and the levels of growth hormone $(\mathrm{GH})$ and insulin-like growth factor binding protein-3 (IGFBP-3) was investigated. From April 2014 to June 2017, 122 children with dwarfism who were treated in The Affiliated Wuxi No. 2 People's Hospital of Nanjing Medical University and The First Affiliated Hospital of Xinxiang Medical University were selected as the experimental group, and 51 normal children as the control group. The basic information was recorded in detail; serum GH and IGFBP-3 levels were measured using an arginine stimulation test and an insulin hypoglycemia stimulation test, respectively. According to the peak of $\mathrm{GH}$ in the experimental group, there were 65 cases of growth hormone deficiency (GHD) and 57 cases of idiopathic short stature (ISS). The expression levels of IGF-1 of the serum in the experimental and control group were detected by chemiluminescence immunoassay (CLIA). The correlation between IGF-1 and GH, IGF-1 and IGFBP-3 was analyzed. The expression level of serum IGF-1 in GHD group was significantly lower than that in the ISS group $(\mathrm{P}<0.05)$. The expression level of serum IGF-1 in GHD group was significantly lower than that in the control group $(\mathrm{P}<0.05)$. The expression level of serum IGF-1 in ISS group was significantly lower than that in the control group $(\mathrm{P}<0.05)$. The results of partial correlation studies showed that IGF-1 is positively correlated with GH and IGFBP-3. Detection of GH and IGFBP-3 are important for the early diagnosis and comprehensive evaluation of children with dwarfism, and also in the detection of IGF-1 can reflect the therapeutic effect of dwarfism on recombinant human growth hormone (rhGH) treatment, which is worthy of application in clinics.
\end{abstract}

Correspondence to: Dr Xiaoli Ge, Department of Pediatrics, The Affiliated Wuxi No. 2 People's Hospital of Nanjing Medical University, 68 Zhongshan Road, Wuxi, Jiangsu 214000, P.R. China E-mail: dac82w@163.com

Key words: insulin-like growth factor-1, insulin-like growth factor binding protein-3, dwarfism, growth hormone, recombinant human growth hormone

\section{Introduction}

Dwarfism is one of the common diseases in pediatrics. Different measures are taken according to the causes, such as increase of the physical activities, monitoring of the height regularly and increase of nutritional supplementation. Short-term treatment is very important in early treatment, with the combination of medical treatment, scientific eating habits and exercise habits, which can promote normal height growth (1). Growth retardation phenomenon has a certain impact on people's self-confidence, work, living conditions and marriage $(2,3)$. Studies have shown (4) that people with dwarfism account for $3 \%$ of the total population, and dwarfism relates to $>400$ genetic and endocrine factors. Also it is related to malnutrition, psychological factors, living environment and economic situation. However, some children with dwarfism have sufficient hormone secretion and no problems, such as malnutrition or mental illness. This is the idiopathic short stature (ISS) (5). ISS is the most common type of dwarfism, accounting for $\sim 60-80 \%$ (6). Currently, the pathogenesis of ISS is unclear. However, as research continues, studies have confirmed the safety and effectiveness of recombinant human growth hormone (rhGH) in the treatment of ISS (7). Clinically, disease analysis and growth hormone $(\mathrm{GH})$ stimulation tests are used to distinguish whether the dwarfism is caused by growth hormone deficiency (GHD). Insulin-like growth factor-1 (IGF-1) is a mediator of $\mathrm{GH}$ that promotes human growth. Its role is to act directly on the GH receptor. The synthesis of IGF-1 is also affected by the regulation of $\mathrm{GH}$ and nutritional status $(8,9)$. The main mechanism of IGF-1 production is to promote the secretion of IGF-1 by generating a stimulation effect by exogenous $\mathrm{GH}$, and to stimulate the $\mathrm{GH}$ according to the reaction condition and make corresponding evaluation $(10,11)$. In normal serum, the IGF-1 binding protein is mainly insulin-like growth factor binding protein-3 (IGFBP-3), and the GH stimulates IGF-1 and promotes the production of IGFBP-3. Both levels of serum are very inconsistent, both circadian rhythm and non-pulsed secretion can reflect the state of hormone secretion (12). In this study, serum GH and IGFBP-3 levels were measured by stimulation tests, and the GHD and ISS groups were determined according to the GH peak in the experimental group. IGF-1 levels of the serum in the experimental and control group were detected by chemiluminescence immunoassay (CLIA) and the correlation between IGF-1 and GH, IGF-1 and IGFBP-3 was analyzed. 
Table I. General clinical baseline data [n (\%)].

\begin{tabular}{|c|c|c|c|c|c|}
\hline \multirow[b]{2}{*}{ Groups } & \multicolumn{2}{|c|}{ Experimental group } & \multirow[b]{2}{*}{ Control group $(\mathrm{n}=51)$} & \multirow[b]{2}{*}{ F value } & \multirow[b]{2}{*}{ P-value } \\
\hline & GHD group $(n=65)$ & ISS group $(\mathrm{n}=57)$ & & & \\
\hline Sex & & & & 0.327 & 0.849 \\
\hline Male & $31(47.69)$ & $29(50.88)$ & $27(52.94)$ & & \\
\hline Female & $34(52.31)$ & $28(49.12)$ & $24(47.06)$ & & \\
\hline Age (years) & & & & 0.020 & 0.990 \\
\hline$<6$ & $30(46.15)$ & $27(47.37)$ & $24(47.06)$ & & \\
\hline$\geq 6$ & $35(53.85)$ & $30(52.63)$ & $27(52.94)$ & & \\
\hline Height (cm) & & & & 2.116 & 0.347 \\
\hline$<100$ & $45(69.23)$ & $42(73.68)$ & $31(60.78)$ & & \\
\hline$\geq 100$ & $20(30.77)$ & $15(26.32)$ & $20(39.22)$ & & \\
\hline Weight (kg) & & & & 1.631 & 0.442 \\
\hline$<20$ & $36(55.38)$ & $26(45.61)$ & $23(45.10)$ & & \\
\hline$\geq 20$ & $29(44.62)$ & $31(54.39)$ & $28(54.90)$ & & \\
\hline Birth weight (kg) & & & & 0.858 & 0.651 \\
\hline$<2$ & $23(35.38)$ & $19(33.33)$ & $14(27.45)$ & & \\
\hline$\geq 2$ & $42(64.62)$ & $38(66.67)$ & $37(72.55)$ & & \\
\hline Paternal height $(\mathrm{cm})$ & & & & 1.171 & 0.557 \\
\hline$<100$ & $33(50.77)$ & $25(43.86)$ & $21(41.18)$ & & \\
\hline$\geq 100$ & $32(49.23)$ & $32(56.14)$ & $30(58.82)$ & & \\
\hline Maternal height $(\mathrm{cm})$ & & & & 0.282 & 0.869 \\
\hline$<100$ & $31(47.69)$ & $27(47.37)$ & $22(43.14)$ & & \\
\hline$\geq 100$ & $34(52.31)$ & $30(52.63)$ & $29(56.86)$ & & \\
\hline Bone age (years) & & & & 3.670 & 0.160 \\
\hline$<7$ & $35(53.85)$ & $31(54.39)$ & $23(45.10)$ & & \\
\hline$\geq 7$ & $20(30.77)$ & $26(45.61)$ & $28(54.90)$ & & \\
\hline
\end{tabular}

GHD, growth hormone deficiency; ISS, idiopathic short stature.

\section{Patients and methods}

General information. From April 2014 to June 2017, 122 children with dwarfism were treated in the Affiliated Wuxi No. 2 People's Hospital of Nanjing Medical University (Wuxi, China) and The First Affiliated Hospital of Xinxiang Medical University (Xinxiang, China). Among them, 122 children with dwarfism were selected as the experimental group and 51 normal children as the control group. According to the peak of GH in the experimental group, there were 65 cases of GHD (GHD group) and 57 cases of idiopathic shortness (ISS group). In the GHD group, there were 31 males and 34 females, aged 6-11 years, with an average age of 9.23 years. In the ISS group, there were 29 males and 28 females, aged 5-12 years, with an average age of 8.85 years. In the control group, there were 27 males and 24 females, aged 5-11 years, with an average age of 9.01 years. All patients underwent thyroid function and urine routine examination before the study was carried out. Children with kidney disease, hypothyroidism or other kidney-related diseases were excluded. The study was approved by the Ethics Committees of The Affiliated Wuxi No. 2 People's Hospital of Nanjing Medical University and The First Affiliated Hospital of Xinxiang Medical University. The parents of the child patients who participated in this research were informed of the details of the study before the detection and signed an informed consent. Patients had complete clinical data. There were no significant differences in terms of sex, age, height, weight, birth weight, paternal weight, maternal weight and bone age between the groups $(\mathrm{P}>0.05)$, which suggested that the groups were comparable.

Specimen collection. A total of $4 \mathrm{ml}$ of venous blood were taken in the morning, on an empty stomach and in a quiet state, and the test was performed in time to separate the serum. The serum GH and IGFBP-3 levels were measured by arginine and insulin hypoglycemia stimulation tests, respectively.

Method. IGF-1 was detected by the method of immunochemiluminescence, using DPC Immulite 2000 chemiluminescence instrument (Siemens AG, Munich, Germany).

GH excitation test. GH stimulation tests were performed using the arginine stimulation test and the insulin hypoglycemia stimulation test. The serum GH levels were examined at 0,30, 


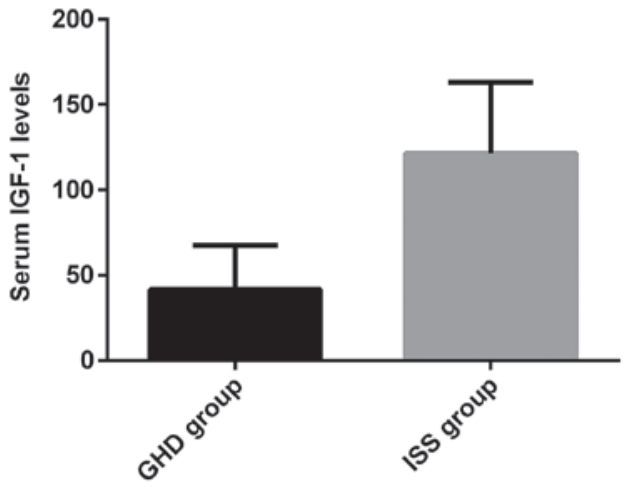

Figure 1. Comparison of serum IGF-1 levels between GHD and ISS group. The expression level of serum IGF-1 in GHD group was significantly lower than that in the ISS group, and there was a significant difference between them $(t=12.92, \mathrm{P}<0.05)$. IGF-1, insulin-like growth factor-1; GHD, growth hormone deficiency; ISS, idiopathic short stature.

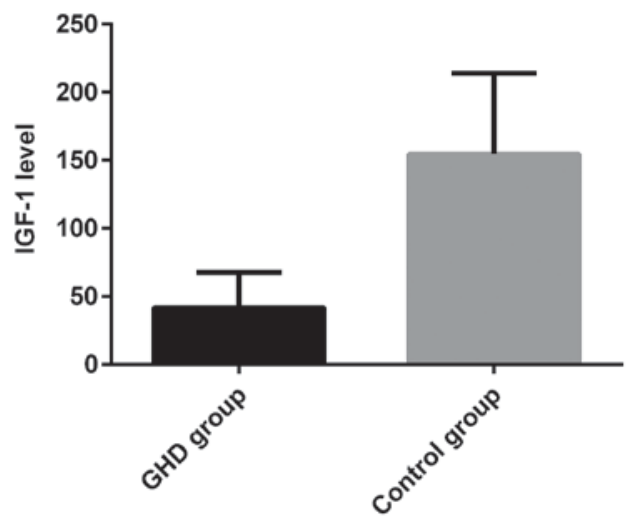

Figure 2. Comparison of serum IGF-1 levels between GHD and control group. The expression level of serum IGF-1 in GHD group was significantly lower than that in the control group, and there was a significant difference between them ( $\mathrm{t}=13.79, \mathrm{P}<0.05)$. IGF-1, insulin-like growth factor-1; GHD, growth hormone deficiency.

60 , and $90 \mathrm{~min}$, respectively. A peak of $\mathrm{GH}<10 \mathrm{ng} / \mathrm{ml}$ denoted GHD, while a peak of GH $>10 \mathrm{ng} / \mathrm{ml}$ denoted ISS.

Statistical analysis. SPSS 17.0 software (Beijing Strong-Vinda Information Technology Co., Ltd., Beijing, China) was used for statistical analysis. The enumeration data were expressed as $\mathrm{n}(\%)$. The comparison among groups was tested by $\mathrm{F}$ test, and the correlation analysis was conducted by partial correlation analysis. $\mathrm{P}<0.05$ was considered to indicate a statistically significant difference.

\section{Results}

General information. There was no significant difference between the experimental and control group in terms of sex, age, height, weight, birth weight, paternal weight, maternal weight or bone age $(\mathrm{P}>0.05)$ (Table I).

Comparison of serum IGF-1 expression levels between the experimental and control group. The levels of serum IGF-1 in GHD and ISS group were $41.75 \pm 25.75$ and $121.53 \pm 41.51$, respectively. The expression of serum IGF-1 in GHD group

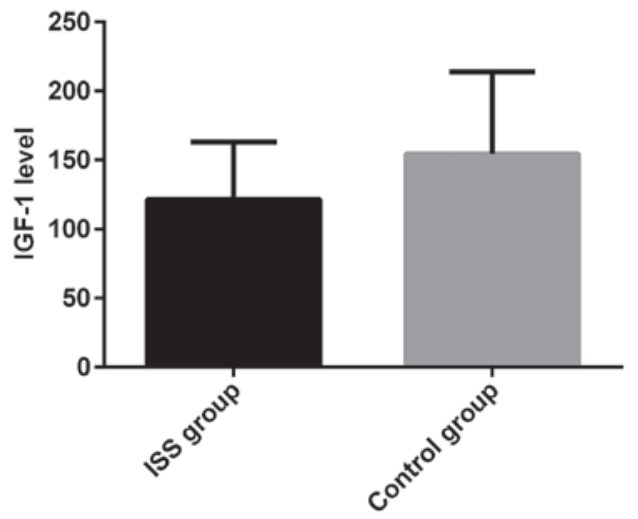

Figure 3. Comparison of serum IGF-1 levels between ISS and control group. The expression level of serum IGF-1 in ISS group was significantly lower than that in the control group, and there was a significant difference between them $(\mathrm{t}=3.38, \mathrm{P}<0.05)$. IGF-1, insulin-like growth factor-1; ISS, idiopathic short stature.

was significantly lower than that in the ISS group. There was significant difference between the groups $(\mathrm{t}=12.92, \mathrm{P}<0.05)$. The expression levels of serum IGF-1 in GHD and control group were $41.75 \pm 25.75$ and $154.54 \pm 59.27$, respectively. The expression level of serum IGF-1 in GHD group was significantly lower than that in the control group, and the difference between the groups was statistically significant $(t=13.79$, $\mathrm{P}<0.05)$. The expression level of serum IGF-1 in the ISS and control group were $121.53 \pm 41.51$ and $154.54 \pm 59.27$, respectively. The expression level of serum IGF-1 in ISS group was lower than that in the control group, and the difference was statistically significant $(\mathrm{t}=3.38, \mathrm{P}<0.05)$ (Figs. 1-3).

Correlation between IGF-1 and GH in dwarfism. Partial correlation analysis showed that the expression levels of $\mathrm{GH}$ and IGF-1 were positively correlated in the serum of patients with dwarfism ( $r=0.974, \mathrm{P}<0.001)$ (Fig. 4).

Correlation between IGF-1 and IGFBP-3 in dwarfism. Partial correlation analysis showed that the expression levels of IGFBP-3 and IGF-1 were positively correlated in the serum of patients with dwarfism $(\mathrm{r}=0.970, \mathrm{P}<0.001)$ (Fig. 5).

\section{Discussion}

Dwarfism in children affects the growth and development of children's height and has an impact on their study and living abilities. Thus, it causes mental and physical burden. Studies have shown that the incidence of dwarfism in children is related to a certain genetic predisposition $(13,14)$. Some studies have reported (15) that children's height is affected by congenital and acquired environmental factors, such as physical exercise, eating habits and quality of life. The causes of dwarfism in children are complicated. They are generally thought to be associated with decreased hormone sensitivity in children or decreased receptor sensitivity, such as growth factors. Maintaining the expression level of IGF-1 is important for promoting the growth and development of children. Moreover, IGF-1 also has a certain effect on the organ function and physiological regulation mechanism of children $(16,17)$. The presence of IGF-1 in human serum is mostly synthesized by the liver, and the expression level is 


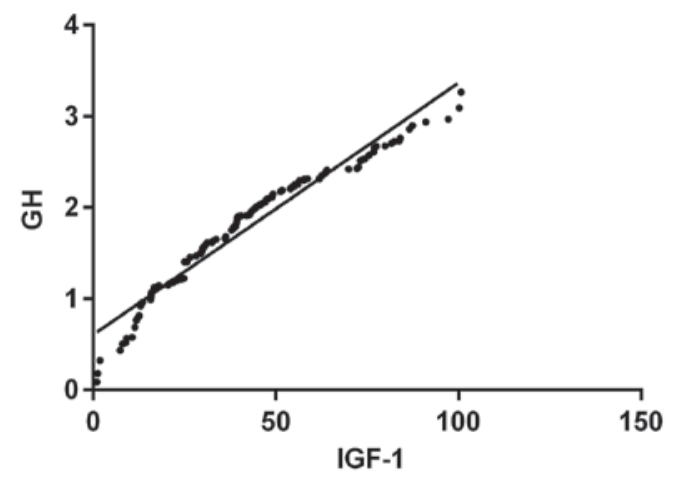

Figure 4. Correlation between GH and IGF-1 in the serum of patients with dwarfism. The results of partial correlation analysis showed that the expression levels of GH and IGF-1 were positively correlated in the serum of patients with dwarfism $(\mathrm{r}=0.974, \mathrm{P}<0.001)$. $\mathrm{GH}$, growth hormone; IGF-1, insulin-like growth factor-1.

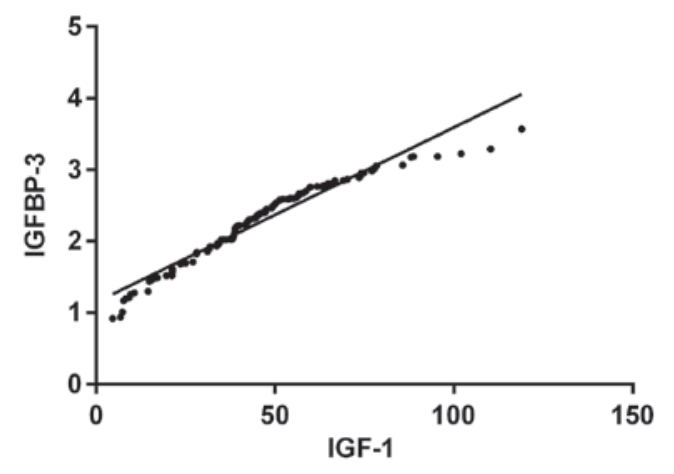

Figure 5. Correlation between IGF-1 and IGFBP-3 in the serum of patients with dwarfism. The results of partial correlation analysis showed that the expression levels of IGFBP-3 and IGF-1 were positively correlated in the serum of patients with dwarfism $(r=0.970, P<0.001)$. IGF-1, insulin-like growth factor-1; IGFBP-3, insulin-like growth factor binding protein-3.

also affected by various factors. The most important factor is $\mathrm{GH}$. After liver GH binds to GH, IGF-1 is secreted, and IGF-1 inhibits GH secretion by negative feedback (18). The IGFBP-3 gene is located in the 7p12-p13 region and is highly conserved. Studies have shown (19) that more than half of the IGFBP-3 levels are genetically determined, but the specific mechanism remains unclear. Polymorphisms in the promoter region of IGFBP-3 gene can change the levels of IGFBP-3 and IGF-1, thereby affecting the development of the disease (20).

Herfs et al (21) have reported that IGF-1 is usually synthesized by the action of $\mathrm{GH}$, and $\mathrm{GH}$ acts on bone through the auxiliary action of IGF-1. IGFBP-3 is the most important insulin-like growth factor, as well as the most powerful one. GH stimulates the synthesis of IGFBP-3 while producing IGFBP-3. IGF-1 has significant negative effects on $\mathrm{GH}$, and IGF-1 is the main regulator of GH. In this study, the differences between the experimental and control group in terms of sex, age, height, weight, birth weight, paternal weight, maternal weight, and bone age were not statistically significant. The expression level of IGF-1 in GHD group was lower than that in ISS group, and there was a significant difference between the groups $(\mathrm{P}<0.05)$. The expression level of IGF-1 in the GHD group was significantly lower than that in the control group $(\mathrm{P}<0.05)$; and the expression level of serum IGF-1 in the ISS group was significantly lower than that in the control group $(\mathrm{P}<0.05)$. This suggests that IGF-1 has an important significance in the diagnosis of children with dwarfism.

Related studies have shown that the levels of serum IGF-1 in patients with GHD are significantly lower than in normal children, and IGF-1 detection can be used as an effective indicator for screening growth retardation caused by abnormal GH-IGF axis (22), which is consistent with the view of this study. However, this study has obvious advantages in terms of number of subjects investigated and the results are more convincing. We also analyzed the correlation between GH and IGF-1 in the serum of patients with dwarfism, and partial correlation analysis showed that GH and IGF-1 are positively correlated $(\mathrm{P}<0.001)$. Therefore, it is speculated that the expression levels of GH and IGF-1 are closely related in dwarfism. Previous studies (23) have shown that the pathogenic mechanisms of ISS and GHD are different. The GHD is caused by various factors leading to decreased GH levels, resulting in limited growth and development. Currently, regarding the treatment of rhGH, some scholars have compared the treatment of ISS and GHF and found that the standard deviation of the mean height of children with GHF has been significantly improved (24). However, another study has suggested that when comparing the treatment of ISS and GHD, the rhGH treatment can improve the height of children with ISS once they enter adulthood (25).

Lanes et al (26) have confirmed that $\mathrm{rhGH}$ treatment of ISS and GHD is a safe and effective treatment. These studies are also consistent with our results. Finally, we analyzed the correlation between IGF-1 and IGFBP-3 in the serum of patients with dwarfism, and the results showed that the expression levels of IGFBP-3 and IGF-1 are positively correlated $(\mathrm{P}<0.001)$. Therefore, it is speculated that the expression levels of IGFBP-3 and IGF-1 are closely related in dwarfism. Rasat et al (27) have shown that the detection of both IGFBP-3 and IGF-1 are better than IGF-1 for the diagnosis of dwarfism. This shows that the joint diagnosis can improve the accuracy.

However, there are certain limitations in our study, as we did not evaluate the prognosis and survival rate of dwarfism, and the clinical and pathological features of IGFBP-3 and IGF-1 in patients with dwarfism were not studied in depth. In the future, we aim to improve our research based on the patients' data in the experimental group, follow-up patients regularly and evaluate the results of this analysis.

In summary, the detection of IGF-1 and IGFBP-3 is important for the early diagnosis and comprehensive evaluation of children with dwarfism. IGF-1 can reflect the therapeutic effect of dwarfism on rhGH, which is worthy of clinical application.

\section{Acknowledgements}

Not applicable.

\section{Funding}

No funding was received.

\section{Availability of data and materials}

The datasets used and/or analyzed during the present study are available from the corresponding author on reasonable request. 


\section{Authors' contributions}

YW and HZ conceived and designed the study and were responsible for the statistical analysis. YW wrote the manuscript. MC acquired the general data of the patients. LK and MC assisted with the specimen collection. XG and YW performed the $\mathrm{GH}$ excitation test. All authors read and approved the final manuscript.

\section{Ethics approval and consent to participate}

The study was approved by the Ethics Committees of The Affiliated Wuxi No. 2 People's Hospital of Nanjing Medical University (Wuxi, China) and The Fisrt Affiliated Hospital of Xinxiang Medical University (Xinxiang, China). The parents of the child patients who participated in this research signed an informed consent, and the patients had complete clinical data.

\section{Patient consent for publication}

Not applicable.

\section{Competing interests}

The authors declare that they have no competing interests.

\section{References}

1. Toumba M, Kokotsis V, Savva SC and Skordis N: Expensive therapies in children: Benefit versus cost of combined treatment of recombinant human growth hormone and gonadotropin-releasing hormone analogue in girls with poor height potential. J Pediatr Endocrinol Metab 27: 311-316, 2014.

2. Sandberg DE and Gardner M: Short stature: Is it a psychosocial problem and does changing height matter? Pediatr Clin North Am 62: 963-982, 2015.

3. Gohlke BC and Stanhope R: Final height in psychosocial short stature: Is there complete catch-up? Acta Paediatr 91: 961-965, 2002.

4. Baten J, Pelger I and Twrdek L: The anthropometric history of Argentina, Brazil and Peru during the 19th and early 20th century. Econ Hum Biol 7: 319-333, 2009.

5. Quitmann JH, Bullinger M, Sommer R, Rohenkohl AC and Bernardino Da Silva NM: Associations between psychological problems and quality of life in pediatric short stature from patients' and parents' perspectives. PLoS One 11: e0153953, 2016.

6. Cohen LE: Idiopathic short stature: A clinical review. JAMA 311: 1787-1796, 2014.

7. Cuttler L: Safety and efficacy of growth hormone treatment for idiopathic short stature. J Clin Endocrinol Metab 90: 5502-5504, 2005.

8. Bereket A, Turan S, Omar A, Berber M, Ozen A, Akbenlioglu C and Haklar G: Serum IGF-I and IGFBP-3 levels of Turkish children during childhood and adolescence: Establishment of reference ranges with emphasis on puberty. Horm Res 65: 96-105, 2006.

9. Cappa M, Iughetti L, Loche S, Maghnie M and Vottero A; GeNeSIS National Board on behalf of the GeNeSIS Italian Investigators: Efficacy and safety of growth hormone treatment in children with short stature: The Italian cohort of the GeNeSIS clinical study. J Endocrinol Invest 39: 667-677, 2016.

10. Wit JM and Rekers-Mombarg LT; Dutch Growth Hormone Advisory Group: Final height gain by GH therapy in children with idiopathic short stature is dose dependent. J Clin Endocrinol Metab 87: 604-611, 2002

11. Kozuki N, Katz J, Lee AC, Vogel JP, Silveira MF, Sania A, Stevens GA, Cousens S, Caulfield LE, Christian P, et al; Child Health Epidemiology Reference Group Small-for-Gestational-Age/Preterm Birth Working Group: Short maternal stature increases risk of small-for-gestational-age and preterm births in low- and middle-income countries: Individual participant data meta-analysis and population attributable fraction. J Nutr 145: 2542-2550, 2015.
12. Xu S, Gu X, Pan H, Zhu H, Gong F, Li Y and Xing Y: Reference ranges for serum IGF-1 and IGFBP-3 levels in Chinese children during childhood and adolescence. Endocr J 57: 221-228, 2010.

13. Şıklar Z, Kocaay P, Çamtosun E, İsakoca M, Hacıhamdioğlu B, Savaş Erdeve Ş and Berberoğlu M: The effect of recombinant growth hormone treatment in children with idiopathic short stature and low insulin-like growth factor-1 levels. J Clin Res Pediatr Endocrinol 7: 301-306, 2015.

14. Grimberg A, DiVall SA, Polychronakos C, Allen DB, Cohen LE, Quintos JB, Rossi WC, Feudtner C and Murad MH; Drug and Therapeutics Committee and Ethics Committee of the Pediatric Endocrine Society: Guidelines for growth hormone and insulin-like growth factor-I treatment in children and adolescents: growth hormone deficiency, idiopathic short stature, and primary insulin-like growth factor-I deficiency. Horm Res Paediatr 86: 361-397, 2016.

15. Huchko MJ, Leslie H, Maloba M, Zakaras J, Bukusi E and Cohen CR: Outcomes up to 12 months after treatment with loop electrosurgical excision procedure for cervical intraepithelial neoplasia among HIV-infected women. J Acquir Immune Defic Syndr 69: 200-205, 2015.

16. Cengiz P, Bas F, Atalar F, Ucar A, Darendeliler F, Akan G, Tarhan T and Bundak R: Growth hormone/insulin-like growth factor- 1 axis as related to body mass index in patients with idiopathic short stature. J Clin Res Pediatr Endocrinol 5: 13-19, 2013.

17. Kawashima Y, Hakuno F, Okada S, Hotsubo T, Kinoshita T, Fujimoto M, Nishimura R, Fukushima T, Hanaki K, Takahashi S, et al: Familial short stature is associated with a novel dominant- negative heterozygous insulin-like growth factor 1 receptor (IGF1R) mutation. Clin Endocrinol (Oxf) 81: 312-314, 2014.

18. Takeshita H, Fujihara J, Soejima M, Koda Y, Kimura-Kataoka K, Ono R, Yuasa I, Iida R, Ueki M, Nagao M, et al: Confirmation that SNPs in the high mobility group-A2 gene (HMGA2) are associated with adult height in the Japanese population; wide-ranging population survey of height-related SNPs in HMGA2. Electrophoresis 32: 1844-1851, 2011.

19. Teng RJ, Wu TJ and Hsieh FJ: Cord blood level of insulin-like growth factor-1 and IGF binding protein-3 in monochorionic twins. J Formos Med Assoc 114: 359-362, 2015.

20. Kaplan RC, Petersen AK, Chen MH, Teumer A, Glazer NL, Döring A, Lam CS, Friedrich N, Newman A, Müller M, et al: A genome-wide association study identifies novel loci associated with circulating IGF-I and IGFBP-3. Hum Mol Genet 20: 1241-1251, 2011.

21. Herfs M, Somja J, Howitt BE, Suarez-Carmona M, Kustermans G, Hubert P, Doyen J, Goffin F, Kridelka F, Crum CP, et al: Unique recurrence patterns of cervical intraepithelial neoplasia after excision of the squamocolumnar junction. Int J Cancer 136: 1043-1052, 2015

22. Bohé J, Joly MO, Arkouche W, Laville M and Fouque D: Haemodialysis with the biocompatible high permeability AN-69 membrane does not alter plasma insulin-like growth factor-I and insulin-like growth factor binding protein-3. Nephrol Dial Transplant 16: 590-594, 2001.

23. Kim SA, Choe YR, Yang EM and Kim CJ: Comparison of growth hormone treatment in patients with idiopathic short stature and idiopathic growth hormone deficiency. Chonnam Med J 50: 63-66, 2014.

24. Hoffman AR: Treatment of the adult growth hormone deficiency syndrome: Directions for future research. Growth Horm IGF Res 15 (Suppl A): 48-52, 2005.

25. Sotos JF and Tokar NJ: Growth hormone significantly increases the adult height of children with idiopathic short stature: Comparison of subgroups and benefit. Int J Pediatr Endocrinol 2014: 15, 2014.

26. Lanes R, Gunczler P, Esaa S and Weisinger JR: The effect of short- and long-term growth hormone treatment on bone mineral density and bone metabolism of prepubertal children with idiopathic short stature: A 3-year study. Clin Endocrinol (Oxf) 57: 725-730, 2002.

27. Rasat R, Livesey JL, Espiner EA, Abbott GD and Donald RA: IGF-1 and IGFBP-3 screening for disorders of growth hormone secretion. N Z Med J 109: 156-159, 1996.

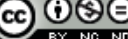

This work is licensed under a Creative Commons Attribution-NonCommercial-NoDerivatives 4.0 International (CC BY-NC-ND 4.0) License. 